\title{
The management of acute venous thromboembolism in clinical practice
}

\author{
Results from the European PREFER in VTE Registry
}

\begin{abstract}
Alexander T. Cohen'; Anselm K. Gitt²; Rupert Bauersachs³, Stefan N. Willich ${ }^{8}$; Peter Bramlage ${ }^{9}$; Giancarlo Agnelli ${ }^{10}$; on behalf of the PREFER in VTE Scientific Steering Committee and the PREFER in VTE Investigators

${ }^{1}$ Guys and St Thomas' Hospitals NHS Foundation Trust, King's College, London, UK; ${ }^{2}$ Herzzentrum Ludwigshafen Med. Klinik B, Ludwigshafen, Germany; ${ }^{3}$ Klinikum Darmstadt $\mathrm{GmbH}$, Darmstadt, Germany; ${ }^{4}$ Center of Thrombosis and Haemostasis, University of Mainz, Mainz, Germany; ${ }^{5}$ Daiichi Sankyo Europe GmbH, Munich, Germany; ${ }^{6} \mathrm{CHU}$ Saint-Etienne Hopital Nord, Saint Etienne Cedex 2, France; ${ }^{7}$ Hospital Universitari Germans Trias i Pujol, Barcelona, Spain; ${ }^{8}$ Charité - Universitätsmedizin Berlin, Germany; ${ }^{9}$ Institute for Pharmacology and Preventive Medicine, Mahlow, Germany; ${ }^{10}$ University of Perugia, Santa Maria della Misericordia Hospital, Perugia, Italy
\end{abstract}

\begin{abstract}
Summary
Venous thromboembolism (VTE) is a significant cause of morbidity and mortality in Europe. Data from real-world registries are necessary, as clinical trials do not represent the full spectrum of VTE patients seen in clinical practice. We aimed to document the epidemiology, management and outcomes of VTE using data from a large, observational database. PREFER in VTE was an international, non-interventional disease registry conducted between January 2013 and July 2015 in primary and secondary care across seven European countries. Consecutive patients with acute VTE were documented and followed up over 12 months. PREFER in VTE included 3,455 patients with a mean age of $60.8 \pm 17.0$ years. Overall, $53.0 \%$ were male. The majority of patients were assessed in the hospital setting as inpatients or outpatients (78.5\%). The diagnosis was deep-vein thrombosis (DVT) in $59.5 \%$ and pulmonary embolism (PE) in $40.5 \%$. The most common
\end{abstract}

\section{Correspondence to:}

Giancarlo Agnelli

Stroke Unit and Division of Cardiovascular Medicine

Department of Internal Medicine

University of Perugia, Santa Maria della Misericordia Hospital

Via Dottori Sant' Andrea delle Fratte, 06126 Perugia, Italy

Tel.: +39075 578 6424, Fax: +39075 5782346

E-mail: agnellig@unipg.it comorbidities were the various types of cardiovascular disease (excluding hypertension; $45.5 \%$ ), hypertension (42.3\%) and dyslipidaemia $(21.1 \%)$. Following the index VTE, a large proportion of patients received initial therapy with heparin $(73.2 \%)$, almost half received a vitamin $\mathrm{K}$ antagonist (48.7\%) and nearly a quarter received a DOAC (24.5\%). Almost a quarter of all presentations were for recurrent VTE, with $>80 \%$ of previous episodes having occurred more than 12 months prior to baseline. In conclusion, PREFER in VTE has provided contemporary insights into VTE patients and their real-world management, including their baseline characteristics, risk factors, disease history, symptoms and signs, initial therapy and outcomes.

\section{Keywords}

Venous thromboembolism, anticoagulation, registry, direct oral anticoagulants, vitamin $\mathrm{K}$ antagonists

Financial support:

This study was funded by Daiichi Sankyo Europe GmbH, Munich, Germany.

Received: October 18, 2016

Accepted after major revision: March 22, 2017

Epub ahead of print: April 13, 2017

https://doi.org/10.1160/TH16-10-0793

Thromb Haemost 2017; 117: 1326-1337

\section{Introduction}

Deep-vein thrombosis (DVT) and pulmonary embolism (PE; collectively termed venous thromboembolism [VTE]) are major healthcare burdens in Europe. Indeed, VTE is highly prevalent in Europe and is the third most frequent cardiovascular (CV) disease $(1,2)$.

Though data from a number of prior VTE registries have been reported $(3,4)$, a great deal of the available information on the clinical management of VTE is drawn from patients included in clinical trials. Due to complex inclusion and exclusion criteria, these patients are often not representative of the full spectrum of VTE patients. Furthermore, the management of patients with VTE has changed substantially following the introduction of new forms of patient care, such as home treatment, office-based care, and the development of new direct oral anticoagulants (DOACs). The majority of the modifications to management strategies are related to the intrinsic features of the available agents (i.e. larger therapeutic windows removing the necessity for close laboratory monitoring) while others (such as reduced hospital stay) are related to socioeconomic and health economic factors. Considering that neither the degree of implementation nor impact of these amendments appear to have been quantified in a real-world setting, acquisition of contemporary epidemiology data on the management of VTE in Europe is warranted.

Based on this background, we developed an international, noninterventional, prospective disease registry enrolling consecutive VTE patients in both primary and secondary care across different 
countries in Europe (5). The aim was to document the epidemiology, management and outcomes of VTE in a large population that was not limited by stringent clinical trial inclusion/exclusion criteria.

\section{Materials and methods}

PREFER in VTE was a prospective, non-interventional, disease registry including patients from seven European countries (Austria, France, Germany, Italy, Spain, Switzerland and the UK). For the present analysis, Germany (D), Austria (A), and Switzerland $(\mathrm{CH})$ were combined into one region, henceforth referred to as "DACH". Prior to study commencement, the registry protocol was approved by the responsible ethics committees for the participating countries and the relevant hospital-based institutional review boards. All patients enrolled in the registry first provided written informed consent. The design and methods of the PREFER in VTE registry have previously been described (5), though a brief overview of the design, patient inclusion criteria and data collection methods is provided below.

\section{Patients}

Patients eligible for participation in the registry were aged at least 18 years, had symptomatic, objectively confirmed acute VTE (either first-time or recurrent) diagnosed in the last two weeks and were not simultaneously participating in a double blind interventional study. Patients were enrolled between January 2013 and 2014, and those in the present study were followed up through to July 2015.

\section{Data collection}

Baseline data were collected in hospitals (on an in- or outpatient basis) or specialised centres at the time of acute VTE diagnosis. The physician was responsible for the input of data pertaining to the period prior to baseline, which was based on a medical history and examination of the patient's medical records. Follow-up data were collected at 1 month (either during a visit or telephone interview) and at 3, 6 and 12 months via a telephone interview with a medically-trained professional. Specially designed patient diaries were kept by each participant to aid their responses to the interview questions and limit recall bias. Furthermore, clear guidelines were adhered to by interviewers in order to standardise the data collection process across the population.

\section{Definitions}

Acute VTE was defined as either distal or proximal DVT, PE or both. Cancer was defined as being active if patients were receiving ongoing treatment, whereas a history of cancer was applicable to all patients with a cancer diagnosis. Cardiovascular disease was defined as one of the following: documented coronary artery disease or vascular disease (e.g. peripheral artery disease or aortic plaque), transient ischaemic attack (TIA); stroke; congestive heart failure or left ventricular systolic dysfunction. Hypertension was defined as a blood pressure $>130 / 80 \mathrm{mmHg}$ in patients with diabetes, and a SBP $>140 \mathrm{mmHg}$ or diastolic blood pressure (DBP) $>90 \mathrm{mmHg}$ in those without diabetes. Liver disease was considered present in patients with chronic hepatic disease or biochemical evidence of significant hepatic derangement. Renal insufficiency was classed into mild (reduction of the glomerular filtration rate [GFR] to 60-89 $\mathrm{ml} / \mathrm{min}$ with kidney damage), moderate (reduction in GFR to $30-59 \mathrm{ml} / \mathrm{min}$ ), severe (reduction in GFR to $15-29 \mathrm{ml} / \mathrm{min}$ ) or end-stage renal disease.

Bleeding risk was assessed using the HAS-BLED score. In this score, one point is awarded for each of the following, up to a maximum of 9 points: hypertension (systolic blood pressure [SBP] $>160$ $\mathrm{mmHg}$ ); abnormal renal function (transplantation, chronic dialysis, or serum creatinine $\geq 200 \mu \mathrm{mol} / \mathrm{L}$ ); abnormal liver function (chronic hepatic disease or biochemical evidence of significant hepatic derangement); stroke (history of stroke); bleeding history or predisposition (anaemia); labile INR (time in therapeutic range $<60 \%$ ); advanced age (>65 years); concomitant drugs/alcohol (one point for use of antiplatelet agents and/or non-steroidal anti-inflammatory drugs, plus one point for excessive alcohol consumption) (6). To assess bleeding outcomes, the International Society of Thrombosis and Haemostasis (ISTH) definitions of major, clinically relevant non-major (CRNM) and nuisance bleeding were used (7).

\section{Statistics}

All binary and categorical outcomes were expressed as frequencies (percentages). Means and standard deviations (SD) were given for normally distributed numerical variables and medians were used for non-parametric data. For all data presented here, values are based on the number of patients for whom the particular data point was available and fully completed, with corresponding denominators. Missing/unknown values are excluded for each factor. Statistical comparisons were carried out using Chi-square and t-tests, with a p-value $<0.05$ considered significant.

\section{Results}

In the PREFER in VTE registry, 3,464 patients were initially registered. Of these, 3,455 (99.7\%) had evaluable baseline data and were included in the current analysis (Figure 1). In the overall population, the majority were assessed at a hospital (78.6\%) as inpatients or outpatients, with only a small proportion undergoing office-based assessment (21.4\%). The hospital assessment rate was higher for PE than for DVT patients (92.5\% vs $69.1 \%$; $\mathrm{p}<0.0001)$.

\section{Patient characteristics}

For all patients included in the analysis, the total mean age was 60.8 years, $53.0 \%$ were male, the mean body mass index (BMI) was $27.9 \mathrm{~kg} / \mathrm{m}^{2}, 31.5 \%$ had a history of smoking, and $17.3 \%$ a his- 


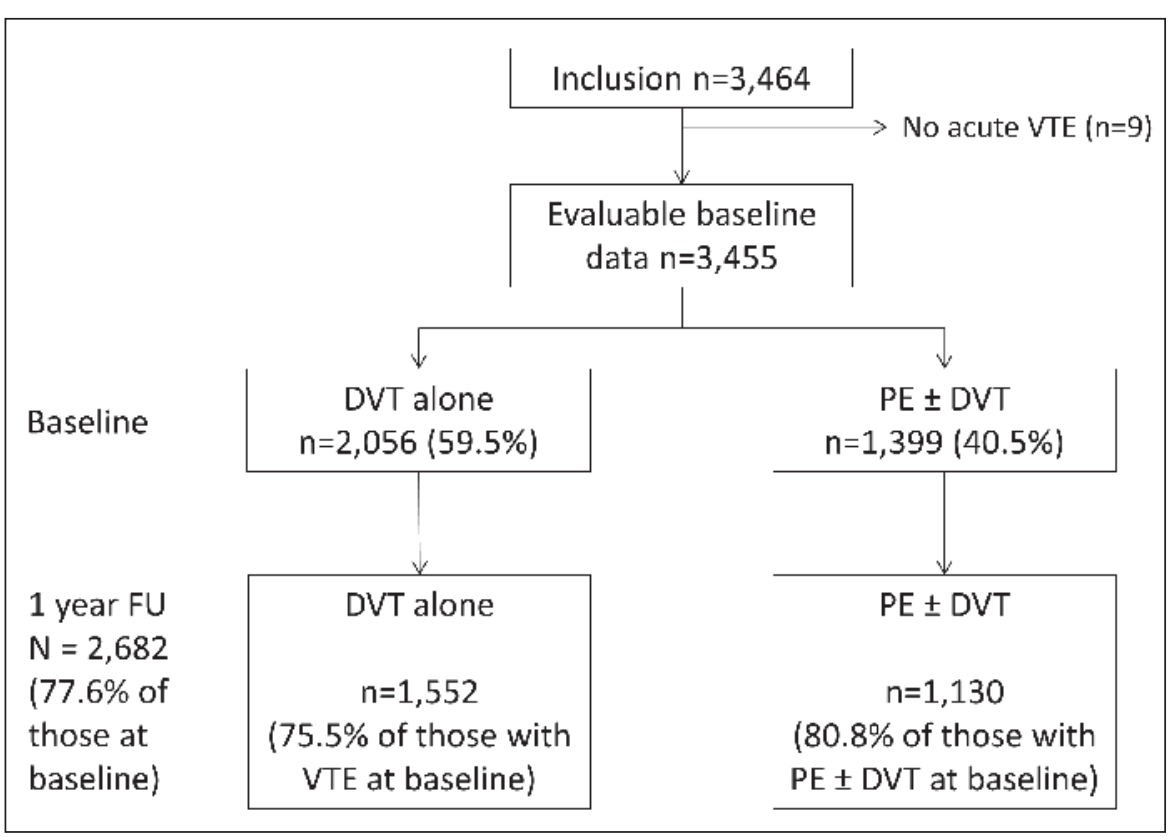

Figure 1: Patient flow. DVT, deep-vein thrombosis; PE, pulmonary embolism. tory of alcohol use (Table 1). No substantial differences between DVT only and PE \pm DVT groups were seen with respect to these demographics. Common comorbidities were CV disease (45.5\%), followed by hypertension (42.3\%), and dyslipidaemia (21.1\%). Each of these comorbidities were more prevalent in patients with $\mathrm{PE} \pm \mathrm{DVT}$ than in those with DVT alone. Before baseline examination, coronary artery disease was present in $3.8 \%$ of all patients, myocardial infarction (MI) and atrial fibrillation (AF) had occurred in $3.3 \%$ and $3.5 \%$, respectively, $2.3 \%$ had suffered a transient ischaemic attack (TIA) and $2.4 \%$ had experienced a stroke. Both $\mathrm{AF}$ and arterial embolization were more common in the PE patients.

\section{VTE-related history and bleeding risk}

A history of varicose veins (20.2\%) and cancer (16.0\%) were prevalent in the study population, with similar proportions seen in the DVT only and PE \pm DV groups (Table 2). Overall, $23.5 \%$ of patients had a history of VTE, applying to a significantly higher proportion of the DVT only compared to the PE \pm DVT group (25.7\% vs $20.2 \%$; $\mathrm{p}=0.0002$ ) (Table 2 ). More DVT than PE \pm DVT patients had experienced a previous DVT episode $(24.5 \%$ vs $15.3 \%$; $\mathrm{p}<0.0001)$, while more PE \pm DVT than DVT patients had experienced a previous PE episode ( $10.0 \%$ vs $5.2 \%$; $\mathrm{p}<0.0001$ ).

The majority of previous DVTs had occurred $>12$ months before baseline (82.7\%) and had resulted in hospitalisation in $42.2 \%$ of cases. Similarly, the majority of previous PE episodes (84.5\%) had also occurred $>12$ months before baseline, though a substantially higher proportion of patients had been hospitalised as a result (84.2\%). Of the patients with a previous DVT episode, $12.3 \%$ were receiving ongoing VTE treatment at baseline. Of patients with a previous PE episode, $13.1 \%$ were receiving ongoing VTE treatment at baseline. This corresponded to only a small proportion of the overall population.

A total of 133 out of 3,451 patients had a history of bleeding events before the baseline visit (Table 3). Of those bleeding events, CRNM bleeding was the most common (51.1\%). Seventeen patients $(12.8 \%)$ had a history of intracerebral bleeding. Based on the HAS-BLED score, bleeding risk was low in the majority of patients (56.7\% had a 0 or 1 points assigned) with no major difference between patients experiencing DVT only (59.4\%) and PE \pm DVT (52.7\%) (Figure 2). Patients with 2 or more points on the HASBLED score made up $>40 \%$ of the cohort. Fourteen percent had a HAS-BLED score of 3 or more points (12.9\% DVT, $15.5 \%$ PE \pm DVT), indicating high risk. The frequencies of individual elements of the HAS-BLED score were also fairly similar between groups.

\section{VTE diagnosis (overall and by type of VTE)}

Upon diagnosis, the most frequent symptoms and signs in relation to DVT were pain $(82.8 \%)$ and the swelling of extremities $(73.3 \%)$ (Table 4). The most frequent symptoms of PE were dyspnea (75.6\%) and chest pain (45.5\%). Only $2.2 \%$ of DVT patients and $6.9 \%$ of PE patients were asymptomatic at the time of diagnosis. The median time between onset of symptoms and first medical contact was 19 hours (h). This was longer for the DVT population $(48 \mathrm{~h})$ compared to patients suffering from PE $(7 \mathrm{~h})$.

In the DVT population, the majority of patients were diagnosed using ultrasound ( $98.6 \%$ ), with a median of 4 days between symptom onset and ultrasound diagnosis. CT/MRI was used for $1.9 \%$ of diagnoses, and venography for $1.6 \%$.

In the PE population the majority of patients were diagnosed using CT/MRI (81.7\%) with a median delay of 2 days between onset of symptoms and CT/MRI diagnosis. A further $17.5 \%$ were diagnosed using echocardiography, and less than $10 \%$ were diag- 
Table 1: Patient characteristics at baseline.

\begin{tabular}{|c|c|c|c|c|}
\hline & $\begin{array}{l}\text { Total } \\
(\mathrm{N}=3,455)\end{array}$ & $\begin{array}{l}\text { DVT only } \\
(n=2,056)\end{array}$ & $\begin{array}{l}P E \pm \text { DVT } \\
(n=1,399)\end{array}$ & $\begin{array}{l}\text { P-value } \\
\text { PE } \pm \text { DVT vs } \\
\text { DVT only }\end{array}$ \\
\hline Age, years & $60.8 \pm 16.97$ & $59.8 \pm 16.84$ & $62.3 \pm 17.05$ & 0.60 \\
\hline Male gender & $1832 / 3455(53.0)$ & $1088 / 2056(52.9)$ & $744 / 1399(53.2)$ & 0.88 \\
\hline $\mathrm{BMI}, \mathrm{kg} / \mathrm{m}^{2}$ & $27.9 \pm 5.57$ & $27.7 \pm 5.28$ & $28.2 \pm 5.95$ & $<0.0001$ \\
\hline Smoking history & $1083 / 3443(31.5)$ & $621 / 2049(30.3)$ & $462 / 1394(33.1)$ & 0.08 \\
\hline Current smoker & $469 / 1080(43.4)$ & $303 / 618(49.0)$ & $166 / 462(35.9)$ & $<0.0001$ \\
\hline Alcohol use & $594 / 3443(17.3)$ & $377 / 2050(18.4)$ & $217 / 1393(15.6)$ & 0.03 \\
\hline \multicolumn{5}{|l|}{ Comorbidities } \\
\hline Hypertension & $1461 / 3451(42.3)$ & $814 / 2053(39.6)$ & $647 / 1398(46.3)$ & 0.0001 \\
\hline Vascular disease (PAD, aorticplaque) & $211 / 3451(6.1)$ & $112 / 2054(5.5)$ & 99/1397 (7.1) & 0.05 \\
\hline Dyslipidaemia & $729 / 3451(21.1)$ & $360 / 2054(17.5)$ & $369 / 1397(26.4)$ & $<0.0001$ \\
\hline Diabetes & $356 / 3451(10.3)$ & $200 / 2053(9.7)$ & $156 / 1398(11.2)$ & 0.18 \\
\hline $\begin{array}{l}\text { Cardiovascular disease(excluding } \\
\text { hypertension) }\end{array}$ & $1571 / 3449(45.5)$ & $871 / 2051(42.5)$ & 700/1398 (50.1) & 0.0001 \\
\hline Renal disease & $213 / 3447(6.2)$ & $123 / 2049(6.0)$ & $90 / 1398(6.4)$ & 0.60 \\
\hline GFR $60-89 \mathrm{ml} / \mathrm{min}$ & $67 / 212(31.6)$ & $40 / 123(32.5)$ & $27 / 89(30.3)$ & \\
\hline GFR $30-59 \mathrm{ml} / \mathrm{min}$ & $103 / 212(48.6)$ & $61 / 123(49.6)$ & $42 / 89(47.2)$ & \\
\hline GFR $15-29 \mathrm{ml} / \mathrm{min}$ & $35 / 212(16.5)$ & $17 / 123(13.8)$ & $18 / 89(20.2)$ & \\
\hline $\begin{array}{l}\text { ESRD (GFR }<15 \mathrm{ml} / \mathrm{min} / \\
\text { permanent renal replacement therapy) }\end{array}$ & $7 / 212(3.3)$ & $5 / 123(4.1)$ & $2 / 89(2.2)$ & \\
\hline Liver disease & $89 / 3451(2.6)$ & $53 / 2053(2.6)$ & $36 / 1398(2.6)$ & 0.99 \\
\hline \multicolumn{5}{|l|}{ History of cardiovascular events } \\
\hline Myocardial infarction & $115 / 3450(3.3)$ & $63 / 2052(3.1)$ & $52 / 1398(3.7)$ & 0.30 \\
\hline Coronary artery disease & $131 / 3451(3.8)$ & $78 / 2054(3.8)$ & $53 / 1397(3.8)$ & 0.996 \\
\hline Percutaneous coronary intervention & $71 / 3450(2.1)$ & $37 / 2053(1.8)$ & 34/1397 (2.4) & 0.20 \\
\hline Coronary artery bypass graft & $28 / 3450(0.8)$ & $18 / 2052(0.9)$ & $10 / 1398(0.7)$ & 0.60 \\
\hline Atrial fibrillation & $120 / 3451(3.5)$ & $54 / 2053(2.6)$ & $66 / 1398(4.7)$ & 0.001 \\
\hline Stroke & $83 / 3451(2.4)$ & $45 / 2053(2.2)$ & $38 / 1398(2.7)$ & 0.32 \\
\hline Transient ischaemic attacks & $78 / 3451(2.3)$ & $41 / 2053(2.0)$ & $37 / 1398(2.6)$ & 0.21 \\
\hline Arterial embolization & 29/3452 (0.8) & $8 / 2054(0.4)$ & $21 / 1398(1.5)$ & 0.0004 \\
\hline Cardiac valve replacement & $13 / 3451(0.4)$ & $8 / 2054(0.4)$ & 5/1397 (0.4) & 0.88 \\
\hline
\end{tabular}

Continuous data are given as mean $\pm \mathrm{SD}$, categorical data are given as $\mathrm{n} / \mathrm{N}(\%)$; BMI, body mass index; DVT, deep vein thrombosis; $\mathrm{PE}$, pulmonary embolism; $\mathrm{SD}$, standard deviation; GFR, glomerular filtration rate; ESRD, end-stage renal disease. nosed using perfusion scan, pulmonary angiography, venous study or ventilation scan.

\section{VTE treatment strategy}

At baseline, the majority of patients in the overall population (73.2\%) were documented as receiving heparin, with a significant number receiving vitamin $\mathrm{K}$ antagonists (VKAs, $49.3 \%$ ) and DOACs (24.9\%). In general, a greater proportion of $\mathrm{PE} \pm \mathrm{DVT}$ pa- tients appeared to be taking heparin and VKAs than patients with DVT alone (84.9\% and $58.0 \%$, vs $65.2 \%$ and $43.3 \%$, respectively), while the opposite was true for DOACs $(21.5 \%$ vs $27.2 \%$, respectively) (Figure 3A). For the subset of countries in which DOACs were launched at study initiation, the aforementioned trend was also evident (Figure 3B). However, the proportion of patients taking DOACs was generally higher than the overall population for both study groups $(42.1 \%$ of DVT patients and $35.9 \%$ of PE \pm DVT patients) and fewer patients were receiving heparin in the 


\begin{tabular}{|c|c|c|c|c|}
\hline & $\begin{array}{l}\text { Total } \\
(\mathrm{N}=3,455)\end{array}$ & $\begin{array}{l}\text { DVT only } \\
(n=2,056)\end{array}$ & $\begin{array}{l}P E \pm D V T \\
(n=1,399)\end{array}$ & $\begin{array}{l}p \text {-value PE } \pm \text { DVT } \\
\text { vs. DVT only }\end{array}$ \\
\hline Previous first episode of VTE & $812 / 3453(23.5)$ & $529 / 2055(25.7)$ & $283 / 1398(20.2)$ & 0.0002 \\
\hline Previous episode of DVT & $718 / 3452(20.8)$ & $504 / 2055(24.5)$ & 214/1397 (15.3) & $<0.0001$ \\
\hline Previous episode of PE & $246 / 3448(7.1)$ & $106 / 2053(5.2)$ & $140 / 1395(10.0)$ & $<0.0001$ \\
\hline Varicose veins & $699 / 3453(20.2)$ & $455 / 2055(22.1)$ & 244/1398 (17.5) & 0.0008 \\
\hline Chronic venous insufficiency & $538 / 3451(15.6)$ & $340 / 2053(16.6)$ & $198 / 1398(14.2)$ & 0.06 \\
\hline History of cancer & $552 / 3451(16.0)$ & $323 / 2054(15.7)$ & 229/1397 (16.4) & 0.60 \\
\hline Active cancer / ongoing treatment & 294/552 (53.3) & $174 / 323(53.9)$ & $120 / 229(52.4)$ & 0.73 \\
\hline $\begin{array}{l}\text { Major surgery or trauma within past } \\
\text { three months }\end{array}$ & $490 / 3453(14.2)$ & 295/2055 (14.4) & 195/1398 (13.9) & 0.74 \\
\hline Bone fractures/soft tissue trauma & $394 / 3450(11.4)$ & $255 / 2053(12.4)$ & 139/1397 (9.9) & 0.03 \\
\hline$>5$ days in bed & $369 / 3451(10.7)$ & 204/2054 (9.9) & 165/1397 (11.8) & 0.08 \\
\hline Chronic respiratory disease & $305 / 3451(8.8)$ & $155 / 2053(7.5)$ & $150 / 1398(10.7)$ & 0.001 \\
\hline Arthritis & 264/3451 (7.6) & $180 / 2053(8.8)$ & $84 / 1398(6.0)$ & 0.003 \\
\hline Known thrombophilic conditions & $226 / 3455(6.5)$ & $155 / 2056(7.5)$ & $71 / 1399(5.1)$ & 0.004 \\
\hline CHF or LVSD & $141 / 3450(4.1)$ & $58 / 2052(2.8)$ & $83 / 1398(5.9)$ & $<0.0001$ \\
\hline Infectious diseases & & & & 0.38 \\
\hline Current & $53 / 3451(1.5)$ & 27/2053 (1.3) & 26/1398 (1.9) & \\
\hline Recent (<3 months) & 65/3451 (1.9) & $41 / 2053(2.0)$ & 24/1398 (1.7) & \\
\hline Lower extremity paralysis & $39 / 3450(1.1)$ & $24 / 2053(1.2)$ & 15/1397 (1.1) & 0.80 \\
\hline Intravenous drug abuse & $11 / 3451(0.3)$ & $9 / 2053(0.4)$ & $2 / 1398(0.1)$ & 0.13 \\
\hline Use of estrogens & $231 / 3452(6.7)$ & $142 / 2054(6.9)$ & 89/1398 (6.4) & 0.53 \\
\hline Females only & 223/1621 (13.8) & 136/967 (14.1) & $87 / 654(13.3)$ & 0.65 \\
\hline Pregnancy† & 29/2697 (1.1) & 24/1533 (1.6) & 5/1164 (0.4) & 0.005 \\
\hline Puerperium & $34 / 3454(1.0)$ & $23 / 2056(1.1)$ & $11 / 1398(0.8)$ & 0.33 \\
\hline \multicolumn{5}{|c|}{$\begin{array}{l}\text { Continuous data are given as mean } \pm S D \text {, categorical data are given as } n / N(\%) \text {; }{ }^{\dagger} \text { Pregnancy was not evaluated from the beginning } \\
\text { but was added to the eCRF at a later stage in the study; BMI, body mass index; DVT, deep vein thrombosis; eCRF, electronic case } \\
\text { report form; PE, pulmonary embolism; SD, standard deviation; VTE, venous thromboembolism; CHF, congestive heart failure; } \\
\text { LVSD, left ventricular systolic dysfunction. }\end{array}$} \\
\hline
\end{tabular}

Table 2: VTE-related risk factors.
DVT group (56.6\%). DOACs were more frequently used in younger patients, those without renal impairment, those at low risk of bleeding, and those with fewer comorbidities (such as diabetes).

At 1-month follow-up, the most prevalent treatment in the overall population was VKA (53.4\%) followed by DOACs (25.4\%) and heparin (18.9\%). In general, a greater proportion of patients with DVT only were taking heparin $(20.2 \%$ vs $16.9 \%)$ or DOACs (27.6\% vs $22.2 \%$ ) compared to PE \pm DVT patients (Figure 3C). Conversely, use of VKAs was more prevalent in the PE \pm DVT group $(61.8 \%$ vs $47.7 \%)$. Again, the same trends were true for countries in which DOACs were launched at study initiation (Figure $3 \mathrm{D})$. However, the proportion of patients treated with DOACs at 1 month was generally higher $(42.6 \%$ and $35.9 \%$ for DVT and $\mathrm{PE} \pm$ DVT groups, respectively) with lower levels of heparin use
(11.6\% and $9.6 \%$ for DVT and PE \pm DVT groups, respectively) than in the corresponding overall groups.

\section{Outcomes 12 months after diagnosis}

Twelve months after diagnosis, 230 patients had died (6.7\% of all those 3,455 patients evaluable at baseline), with similar rates in each group. The PE recurrence rate was generally low (1.3\% overall), the DVT recurrence was higher (3.9\%), largely owing to a higher proportion of DVT only patients experiencing a further DVT event (5.6\% vs $1.7 \%$ for PE \pm DVT patients; $\mathrm{p}<0.0001$ ). Major bleeding occurred in $1.5 \%$, clinically-relevant non-major bleeding (CRNM) in $3.1 \%$, and $4.2 \%$ of patients experienced nuisance bleeding. The only difference between groups was found for CRNM bleeding, with significantly more events occurring in the $\mathrm{PE} \pm$ DVT group (4.2\% vs $2.3 \% ; \mathrm{p}=0.008)$. In those having a fol- 
Table 3: History of bleeding events.

\begin{tabular}{l|l|l|l|l}
\hline & $\begin{array}{l}\text { Total } \\
(\mathbf{N}=3,455)\end{array}$ & $\begin{array}{l}\text { DVT only } \\
(\mathbf{n}=2,056)\end{array}$ & $\begin{array}{l}\text { PE } \pm \text { DVT } \\
(\mathbf{n}=1,399)\end{array}$ & $\begin{array}{l}\text { p-value } \\
\text { PE } \pm \text { DVT vs. DVT only }\end{array}$ \\
\hline Total bleeding & $133 / 3451(3.9)$ & $74 / 2053(3.6)$ & $59 / 1398(4.2)$ & 0.3562 \\
\hline Major bleeding & $36 / 133(27.1)$ & $19 / 74(25.7)$ & $17 / 59(28.8)$ & \\
\hline CRNM bleeding & $68 / 133(51.1)$ & $36 / 74(48.6)$ & $32 / 59(54.2)$ & \\
\hline Nuisance bleeding & $29 / 133(21.8)$ & $19 / 74(25.7)$ & $10 / 59(16.9)$ & \\
\hline Gastrointestinal ${ }^{*}$ & $46 / 133(34.6)$ & $28 / 74(37.8)$ & $18 / 59(30.5)$ & 0.3773 \\
\hline Major bleeding & $12 / 46(26.1)$ & $6 / 28(21.4)$ & $6 / 18(33.3)$ & \\
\hline CRNM bleeding & $23 / 46(50.0)$ & $14 / 28(50.0)$ & $9 / 18(50.0)$ & \\
\hline Nuisance bleeding & $11 / 46(23.9)$ & $8 / 28(28.6)$ & $3 / 18(16.7)$ & 0.0706 \\
\hline Intracerebral & $17 / 133(12.8)$ & $6 / 74(8.1)$ & $11 / 59(18.6)$ & 0.4968 \\
\hline Other & $72 / 133(54.1)$ & $42 / 74(56.8)$ & $30 / 59(50.8)$ & \\
\hline Major bleeding & $16 / 72(22.2)$ & $11 / 42(26.2)$ & $5 / 30(16.7)$ & $18 / 30(60.0)$ \\
\hline CRNM bleeding & $37 / 72(51.4)$ & $19 / 42(45.2)$ & $7 / 30(23.3)$ & \\
\hline Nuisance bleeding & $19 / 72(26.4)$ & $12 / 42(28.6)$ & \\
\hline All data are given as n/N (\%). Multiple reasons could be given for a bleeding event. CRNM, clinically relevant non-major; DVT, \\
deep vein thrombosis; PE, pulmonary embolism; * ${ }^{*}$ patients may have had major and/or CNRM and/or nuisance bleeding.
\end{tabular}

low-up at 12 months available, rates of the individual components of the non-fatal ischaemic events (MI, stroke, TIA and arterial embolism) were extremely low $(\leq 0.5 \%)$ and similar between groups, with the exception of arterial embolisation, which was more prevalent in DVT only patients $(0.5 \%$ vs $0.1 \%$ for PE \pm DVT patients; $\mathrm{p}=0.045$ ) (Table 5). Hospital admission occurred in $17.5 \%$ of patients overall, though greater numbers of PE \pm DVT patients were hospitalised $(21.5 \%)$ compared to DVT only patients $(14.5 \%$; $\mathrm{p}<0.0001)$.

\section{Discussion}

PREFER in VTE was a multinational registry which enrolled over 3,400 patients from seven European countries (5). This multicentre observational database aimed to document VTE clinical management practices and patient outcomes in a wide-ranging sample of sites. These encompassed the spectrum of care from small to specialist primary care practices, small to very large secondary care institutions, and both academic and non-academic sites. PREFER in VTE therefore provides a snapshot of the VTE landscape in Western European countries, including the realworld use of new DOACs.

\section{Patient characteristics}

Patients in the PREFER in VTE registry were generally older (average 61 years) compared to those in recent phase 3 clinical trials (ranging from 55 to 58 years) (8). Conversely, our patients were similar in age or younger than those in inception cohorts (ranging from 62-65 years) $(9,10)$. About half $(53 \%)$ of the PREFER in VTE patients were males compared with $53.0-61 \%$ in clinical trials (8). However, most VTE inception cohorts show higher percentages of females $(9,10)$. This is likely to be partly due to recurrences being more common in males; hence registries and clinical trials contain a higher proportion of male subjects, despite first episodes being more common in females. The mean BMI was 27.9 $\mathrm{kg} / \mathrm{m}^{2}$, similar to what has been described in both clinical trials and inception cohorts $(8,10,11)$. The frequency of risk factors for VTE (such as cancer and a past history of VTE) were similar to clinical trials. Over $23 \%$ of all presentations were for recurrent VTE disease, with the majority of previous episodes $(>80 \%)$ having occurred more than 12 months previously. This is consistent with the view that VTE should be considered as a chronic disease (12). Around $4 \%$ of patients had experienced bleeding prior to developing the index VTE. Given that such patients have not been included in clinical trials, their clinical course is of interest.

Our study may supplement the information already obtained from databases such as the Registro Informatizado de Enfermedad ThromboEmbólica (RIETE) registry, an ongoing, international, prospective registry of acute VTE patients which was started in 2001 (3). In a 2010 analysis including 22,133 patients, the proportion of patients diagnosed with $\mathrm{PE}$ was slightly higher compared to the present study (PE: $47 \%$ vs $41 \%$ ), as was the mean age (65 vs 60 years) (13). This may be for a number of reasons related to differences in study design. Although RIETE also excluded individuals participating in clinical trials, analyses tend to pool data from patients who were diagnosed over a wide range of years (i. e. between 2001 and 2008) (13). Conversely, the present analysis is based on those diagnosed between 2013-2014. This means that patient 


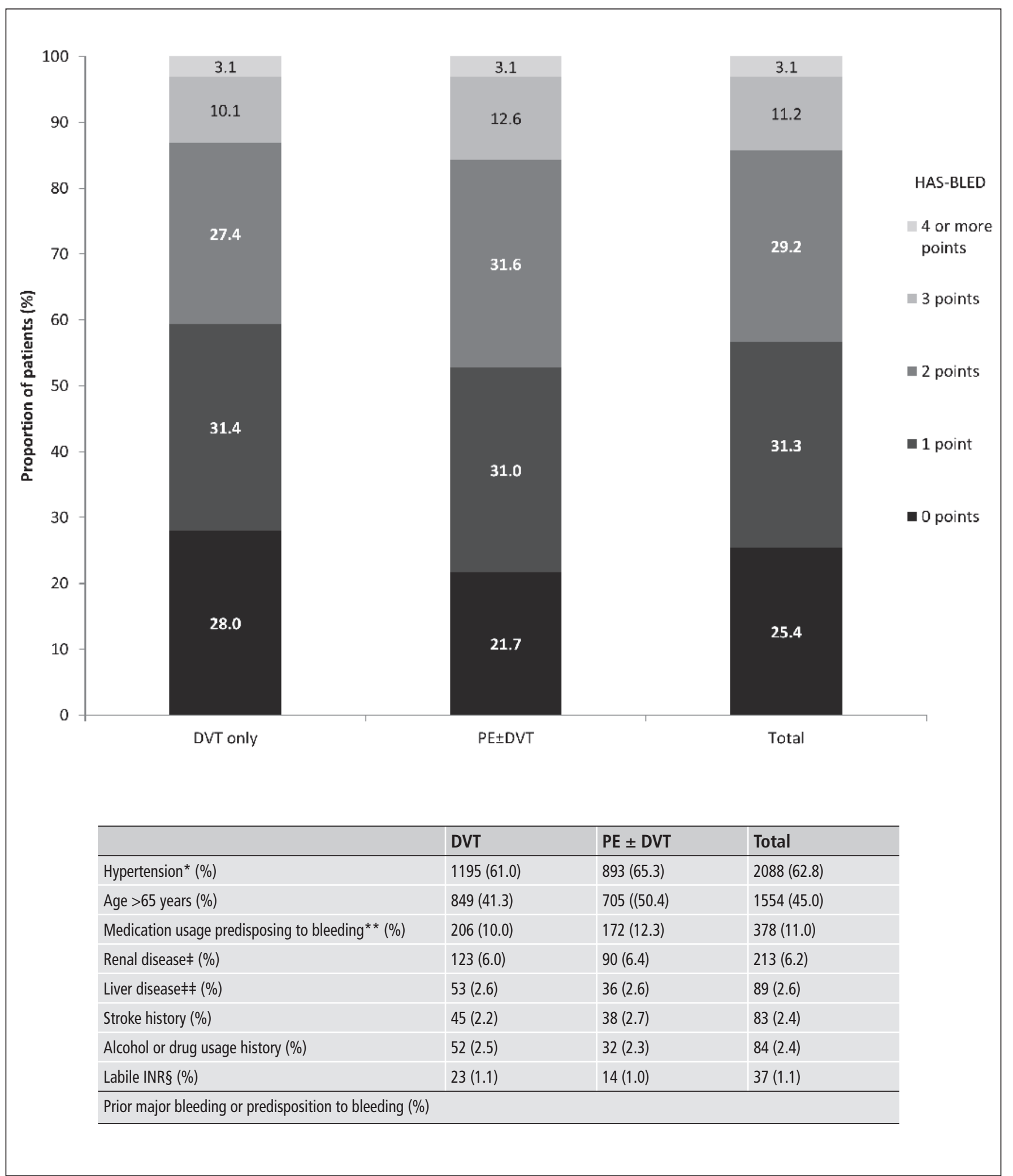

Figure 2: Bleeding risk by type of VTE according to overall HAS-BLED score and individual components $(n=3,292)$. DVT, deep-vein thrombosis; PE, pulmonary embolism. In the HAS-BLED score, one point is assigned for each of the criteria in the lower panel (9 points max). *Systolic blood pressure $>160 \mathrm{mmHg} .{ }^{* *}$ Antiplatelet agents or non-steroidal anti-inflammatory drugs. $\ddagger$ Dialysis, transplant, $\mathrm{Cr}>2.26 \mathrm{mg} / \mathrm{dl}$ or $>200 \mu \mathrm{mol} / \mathrm{l}$. $\ddagger$ Cirrhosis or bilirubin $>2 \times$ normal or AST/ALT/AP $>3 \times$ normal. §time in therapeutic range $<60 \%$. 
numbers are significantly larger in RIETE analyses, though the registry is less geared towards identifying recent shifts in the clinical landscape which may lead to different results. Secondly, the majority of RIETE patients were diagnosed at hospitals in Spain, as well as some in France, Italy, Israel and Brazil. This suggests that sociodemographic biases are likely to be different compared to the present study. Furthermore, hospital diagnosis in the RIETE analysis occurred either following symptomatic emergency room presentation or during hospitalisation for another cause, with both settings indicating more severe disease, high comorbidity levels, and more specialised care. Conversely, the mixture of sites in the PREFER in VTE registry allows for the inclusion of patients diagnosed in a range of settings, and therefore a greater diversity of VTE presentation. Interestingly, despite the aforementioned differences, the reported rates of bleeding events prior to baseline were fairly comparable in RIETE and PREFER in VTE studies (3.0\% vs $3.9 \%$, respectively), suggesting that this may be a consistent factor in VTE patients. Unlike RIETE, our study routinely collected data on factors such as resource utilisation, Quality of Life, and the convenience/burden of anticoagulation treatment, with both general and VTE-specific assessments carried out. Both registries provide valuable and interesting data which may be used to broaden our understanding of the VTE clinical landscape. It will be interesting to compare the results of PREFER in VTE with upcoming European registries, such as the Global Anticoagulant Registry in the Field for Venous Thromboembolism (GARFIELD-VTE) (14).

\section{Symptoms and signs of VTE}

The presentation of symptoms and signs and the methods used for diagnosis in the present study were consistent with previous data $(15,16)$. Asymptomatic PE and DVT made up $6.9 \%$ and $2.2 \%$ of the cohort, respectively. A large difference in the time between clinical onset of symptoms and the time of diagnostic testing was seen between DVT and PE patients, with a shorter duration in the latter population. This is expected, given that $\mathrm{PE}$ is the more serious form of VTE presentation. Patients not usually enrolled in clinical trials were included in the registry, including those with a history of bleeding (3.9\%), women who are pregnant $(1.1 \%)$ or in puerperium $(1.0 \%)$, and intravenous drug users $(0.3 \%)$.

\section{Comorbidity and bleeding risk}

In the present study, we used the HAS-BLED score as the principal measure of bleeding risk. Scores were not seen to vary greatly between DVT and PE patients, with over $40 \%$ of the total population having a value consistent with moderate-to-high risk. Comorbidities were not infrequent; renal impairment and liver disease are important factors to be considered in bleeding risk assessment and occurred in $6.2 \%$ and $2.6 \%$ of patients, respectively. The clinical course of these patients will be of substantial interest, as patients with higher bleeding risk profiles are not typically included in clinical trials.

Although the HAS-BLED score itself was not originally intended for use in assessing VTE patients, having been developed for
Table 4: Symptoms and signs at baseline.

\begin{tabular}{|l|l|}
\hline DVT (n=2,056) & Patients (\%) \\
\hline Pain & $1,703 / 2,056(82.8)$ \\
\hline Swelling of extremity & $1,503 / 2,056(73.3)$ \\
\hline Calf tenderness & $604 / 2,056(29.4)$ \\
\hline Discoloration & $333 / 2,056(16.2)$ \\
\hline Collateral superficial veins & $149 / 2,056(7.2)$ \\
\hline None (asymptomatic) & $45 / 2,056(2.2)$ \\
\hline Other & $120 / 2,056(5.8)$ \\
\hline PE (n=1,399) & \\
\hline Dyspnea & $1058 / 1,399(75.6)$ \\
\hline Chest pain & $637 / 1,399(45.5)$ \\
\hline Cough & $235 / 1,399(16.8)$ \\
\hline Tachycardia & $233 / 1,399(16.7)$ \\
\hline Tachypnea & $226 / 1,399(16.2)$ \\
\hline Syncope & $114 / 1,399(8.1)$ \\
\hline Palpitations & $110 / 1,399(7.9)$ \\
\hline Fever & $109 / 1,399(7.8)$ \\
\hline None (asymptomatic) & $96 / 1,399(6.9)$ \\
\hline Hemoptysis & $48 / 1,399(3.4)$ \\
\hline Cyanosis & $31 / 1,399(2.2)$ \\
\hline Cardiogenic Shock & $21 / 1,399(1.5)$ \\
\hline Other & $106 / 1,399(7.6)$ \\
\hline All data are given as $n / N(\%)$. & \\
\hline & \\
\hline
\end{tabular}

AF patients (6), each of its individual components is a validated risk factor for bleeding in VTE outlined by the 2016 CHEST Guideline and Expert Panel Report on Antithrombotic Therapy for VTE Disease (17). This is with the exception of hypertension, which was recently identified as a predictor for bleeding in 8,240 VTE patients treated with a factor Xa inhibitor or warfarin (18). Indeed, 2016 ESC Guidelines for the Management of AF state that all of the HAS-BLED components should be used for the identification of modifiable risk factors in anticoagulated patients (19). Furthermore, several groups have presented evidence for the use of the HAS-BLED score in VTE populations $(20,21)$, with one reporting HAS-BLED to show the highest predictive value compared to other bleeding-risk assessment scores during the first three months of anticoagulation (21). However, it is worth noting that robust validation of HAS-BLED in a large population is currently lacking. The present study provides some new evidence regarding the distribution of bleeding risk between DVT and PE patients as assessed with the HAS-BLED score, which may be informative to a number of researchers in the field. Nonetheless, there are other risk factors for bleeding in VTE patients which are not accounted for by HAS-BLED, such as cancer, thrombocytopenia, diabetes, recent surgery, and frequent falls (17). These should 

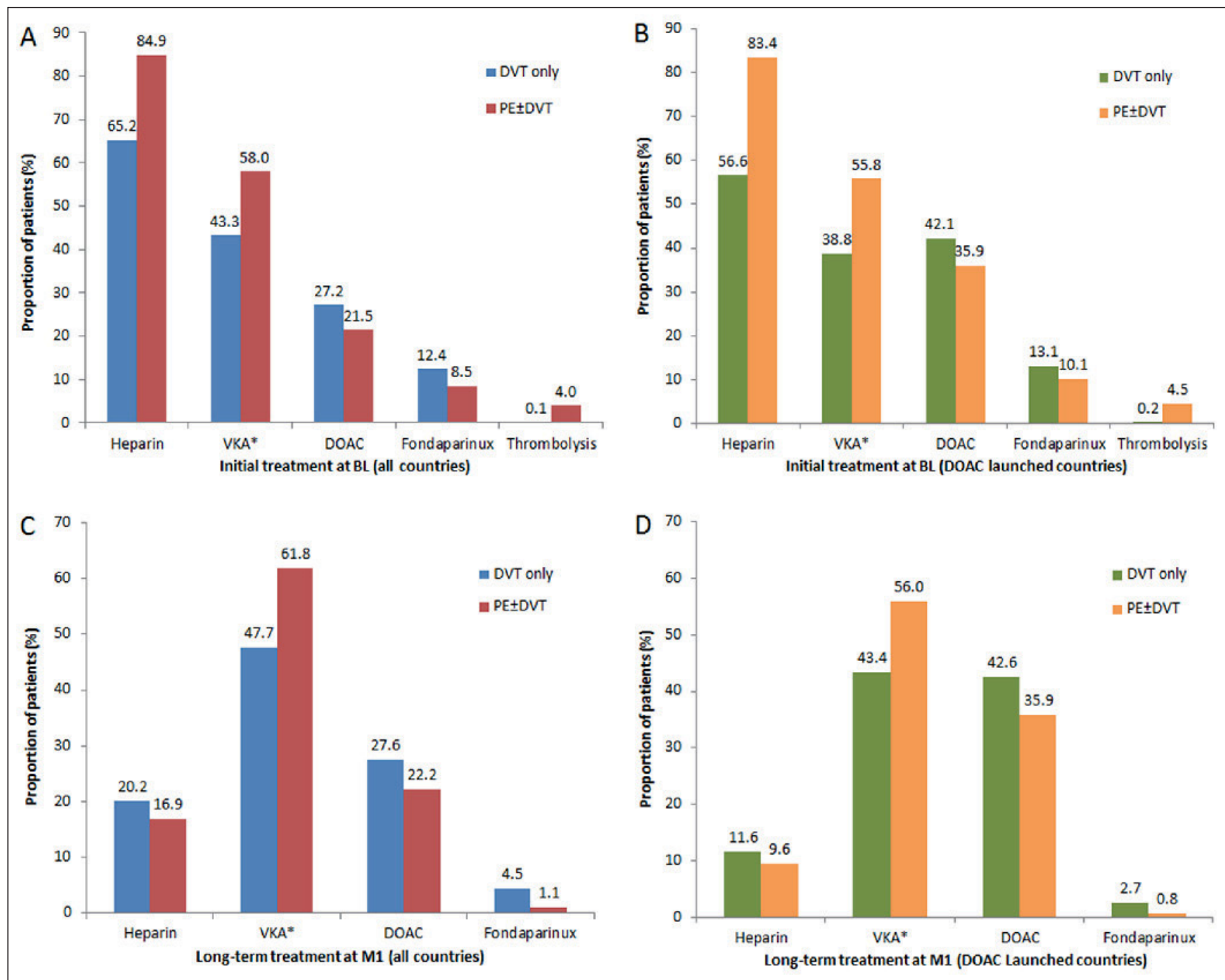

Figure 3: Treatment strategy overall ( $A$ and $C$ ) and within countries with DOAC launch at study commencement (B and D). DOAC, direct oral anticoagulant; DVT, deep-vein thrombosis; PE, pulmonary embolism; VKA, vitamin $\mathrm{K}$ antagonist. Multiple options possible for any one patient.

DOAC launched countries are Germany, Austria, Switzerland, France and UK; Non-DOAC countries are Italy and Spain. *Delay in peak effect after initiation (2-5 days).

also be taken into account when determining risk. Several other scores have been suggested for use in the VTE setting, such as VTE-BLEED, which awards points for six clinical variables (active cancer [2 points]; male gender plus uncontrolled arterial hypertension [ 1 point]; anaemia [1.5 points]; history of bleeding [1.5 points]; age $\geq 60$ years [1.5 points]; and renal dysfunction [1.5 points]) (22). This algorithm, which shares several similarities with the HAS-BLED score, was shown to be strongly predictive for major bleeding in 5,107 VTE patients on stable anticoagulation. Though guidelines currently advocate the presence of 1 (moderate risk) or $\geq 2$ (high risk) out of 11 established risk factors as indicators of a patient's bleeding risk in VTE, further development and validation of existing algorithms such as HAS-BLED and VTEBLEED may replace such recommendations in future.

\section{VTE treatment}

Due to a combination of regulatory statuses, licencing, national and local guidelines and reimbursement at the time of the PREFER in VTE registry, DOAC use varied greatly between countries. Indeed, DOACs were only licensed and reimbursed in $\mathrm{DACH}$, France and the UK. As a result, DOAC data was only fully analysed for these countries. Future analyses of this registry will evaluate the differences in treatment patterns between all included countries.

In the overall registry analysis, almost a quarter of patients were receiving a DOAC at baseline, with little variation between those in the DVT and PE groups (26.8\% vs $21.2 \%$ ). During this early phase of therapy, DOACs were more frequently used in younger patients, those without renal impairment, those at low risk of 
Table 5: Overall outcomes of VTE patients 12 months after diagnosis.

\begin{tabular}{|c|c|c|c|c|}
\hline & $\begin{array}{l}\text { Total } \\
(\mathrm{N}=3,455)\end{array}$ & $\begin{array}{l}\text { DVT only } \\
(n=2,056)\end{array}$ & $\begin{array}{l}P E \pm D V T \\
(n=1,399)\end{array}$ & $\begin{array}{l}\text { p-value } \\
\text { PE } \pm \text { DVT vs. DVT only }\end{array}$ \\
\hline Total mortality * & $230 / 3,455(6.7)$ & $128 / 2,056(6.2)$ & $102 / 1,399(7.3)$ & 0.40 \\
\hline VTE related & $11 / 3,455(0.3)$ & $4 / 2,056(0.2)$ & $7 / 1,399(0.5)$ & 0.14 \\
\hline \multicolumn{5}{|l|}{ Recurrent VTE ** } \\
\hline Non-fatal PE & $30 / 2,333(1.3)$ & $12 / 1,328(0.9)$ & $18 / 1,005$ (1.8) & 0.06 \\
\hline DVT & $92 / 2,341(3.9)$ & 75/1,337 (5.6) & $17 / 1,337(1.7)$ & $<0.0001$ \\
\hline \multicolumn{5}{|l|}{ Bleeding events ** } \\
\hline Major bleeding & $35 / 2,326(1.5)$ & $18 / 1,325$ (1.4) & $17 / 1,001(1.7)$ & 0.51 \\
\hline CNRM & $72 / 2,330(3.1)$ & $30 / 1,327(2.3)$ & $42 / 1,003(4.2)$ & 0.008 \\
\hline Nuisance & $99 / 2,338(4.2)$ & 48/1,334 (3.6) & $51 / 1,004(5.1)$ & 0.08 \\
\hline \multicolumn{5}{|c|}{$\begin{array}{l}\text { Non-fatal ischemic events } \\
\star *\end{array}$} \\
\hline Myocardial infarction & $12 / 2,331(0.5)$ & $7 / 1,329(0.5)$ & $5 / 1,002(0.5)$ & 0.93 \\
\hline Stroke & $5 / 2,330(0.2)$ & $2 / 1,329(0.2)$ & $3 / 1,001(0.3)$ & 0.44 \\
\hline TIA & 9/2,331 (0.4) & $5 / 1,329(0.4)$ & $4 / 1,002(0.4)$ & 0.93 \\
\hline Arterial embolisation & $6 / 2,328(0.3)$ & $5 / 1,000(0.5)$ & $1 / 1,328(0.1)$ & 0.045 \\
\hline Hospital admission ** & 415/2,371 (17.5) & $197 / 1,356(14.5)$ & $218 / 1,015(21.5)$ & $<0.0001$ \\
\hline \multicolumn{5}{|c|}{$\begin{array}{l}\text { All data are given as n/N (\%). Multiple reasons could be given for a bleeding event. CRNM, clinically relevant non-major; DVT, } \\
\text { deep vein thrombosis; PE, pulmonary embolism. *Proportional to all patients recorded at baseline. ** Unless otherwise stated, } \\
\text { each denominator refers to the number of patients with complete data for the relevant variable available at 12-month follow- } \\
\text { up. }\end{array}$} \\
\hline
\end{tabular}

bleeding (according to HAS-BLED), and those with fewer comorbidities (such as diabetes). Concurrently, a similar registry which enrolled AF patients (PREFER in AF) also noted a low use of DOACs in higher-risk patients (23). This may be considered paradoxical, given that the use of DOACs is thought to be more beneficial in higher-risk patients, especially the elderly. A potential explanation for this may be the reluctance of physicians to treat higher-risk patients with the newer agents at the time of the PREFER in VTE registry, likely due to a greater degree of familiarity and confidence using VKAs. Other explanations may include a physicians being unwilling to switch patients from their historical agents, or a lack of understanding of the benefits of new agents. The latter may highlight a need for greater education in this area, especially given that results from pivotal phase III studies suggest that DOACs are as effective as VKAs with improved safety profiles (24-28). Indeed, a meta-analysis comparing the four approved DOACs with warfarin found that all of the DOACs had comparable efficacy with a significantly lower bleeding risk (29). These results and current guidelines suggest that there will be a paradigm shift towards higher DOAC use in the future; however, greater education efforts may be needed for many physicians to become comfortable with DOAC use. Data demonstrating the safe use of a predefined reduced DOAC dose for special subsets of patients (such as those with renal impairment or low bodyweight), may instil greater confidence in the medical professionals prescribing them (24).

\section{Outcomes}

Overall outcomes at 12 months showed event rates that were consistent with previous studies $(2,10,24-28,30,31)$. VTE is a condition associated with significant mortality, and the rates seen were much higher than those found in clinical trial populations (24-28). The higher recurrence rates seen in DVT compared with PE patients may be related to treatment patterns, and this will be examined in future analyses. The rates of major, CRNM and nuisance bleeding and ischemic arterial events were as expected.

\section{Limitations}

While registries are generally more representative of a real-world population and not subject to the stringent inclusion/exclusion criteria restrictions of randomised control trials, a certain degree of sampling bias is unavoidable. Indeed, the majority of patients in the present study were diagnosed with VTE in the hospital setting, with approximately $40 \%$ presenting with PE. "Although hospital diagnosis was not necessarily on an in-patient basis, these findings suggest that the registry may be more representative of those with more severe VTE, limiting generalisability. Furthermore, for countries where diagnosis and management predominantly occur outside of a hospital setting owing to differences in healthcare system structure, our findings may not necessarily be applicable. In addition, very elderly, frail patients are less likely to be able to give informed consent and patients who died suddenly due to PE were 


\section{What is known about this topic?}

- Venous thromboembolism (VTE) represents a significant healthcare burden and is the third most common cardiovascular disease in Europe.

- Much of the data available for the management of VTE are from clinical trials and may therefore not be representative of the full spectrum of VTE patients.

\section{What does this paper add?}

- Analysis of a large European cohort of VTE patients, finding that patients at moderate-to-high risk of bleeding are prevalent amongst those who have a VTE event.

- Almost a quarter of all presentations were for recurrent VTE, with $>80 \%$ of previous episodes having occurred more than 12 months prior to baseline; hence VTE should be considered a chronic disease.

- Uptake of direct oral anticoagulants in Europe has been encouraging, with use particularly common in certain lower-risk subgroups.

not included. Patients under 18 and those actively participating in a randomised, double blind interventional study were also ineligible for enrolment, potentially excluding some younger, healthier subjects from the analysis. Finally, while one of the strengths of this registry is the large variety of sites, which reflects efforts to find a representative spread and account for national differences, confinement of study centres to Western Europe may limit extrapolation of findings to other geographical areas.

Of further note is that patient histories, as with most studies, were based on a retrospective review of medical records. Therefore, some incomplete or inaccurate data may be expected, reducing robustness. Furthermore, the degree of detail recorded may have been insufficient to reliably determine certain variables (such as type and severity of prior bleeding events), and the interpretation of data by physicians may have introduced further subjective bias or inaccuracy. Thus, the reliability of data on events prior to baseline is not fully assured.

In terms of the data collected by telephone interview, a standardised patient diary was employed to record relevant events and limit recall bias. Notwithstanding this, the patient's subjective evaluation of factors (such as bleeding severity) may have influenced the data collected. However, interviews were carried out by professionals with the appropriate medical knowledge and training to ensure standardised definitions were adhered to and further information could be sought from the patient's medical practitioners. Therefore, data are assumed to be valid.

\section{Future perspectives}

Further analyses of the PREFER in VTE registry will provide new insights into the management of VTE patients in Western Europe. Evaluation of the follow-up data of these patients will provide evidence for the changing clinical landscape in VTE treatment. Addi- tionally, patient satisfaction and health-related quality of life will be analysed based on questionnaires completed at baseline and each follow-up.

\section{Conclusions}

The results confirm that VTE is a ubiquitous disorder occurring in many settings, and that in a real-world sample that is unrestricted by the confines of clinical trial inclusion/exclusion criteria, risk factors for bleeding are not uncommon. VTE is also a chronic disease, with initial events often taking place more than a year before subsequent episodes. DOAC use in Europe is fairly common, particularly amongst "lower-risk" patients at present; though this should extend into the higher-risk population as guideline recommendations circulate and physician confidence in DOACs grows. Future analyses from the PREFER in VTE registry are therefore likely to identify changing treatment patterns reflecting recent changes to guideline recommendations.

\section{Acknowledgments}

The authors acknowledge the substantial contribution of Dr. Markus Schwertfeger (Daiichi Sankyo) for his input into the design and setup of the registry. Furthermore we are indebted to all investigators across Austria, France, Germany, Italy, Spain, Switzerland and the UK who have made this registry possible. A full list of the PREFER in VTE investigators has previously been reported and is included in the addendum (5). Editorial assistance during the development of this manuscript was provided by Matthew Bexon of inScience Communications. This assistance was funded by Daiichi Sankyo Europe GmbH, Munich, Germany.

\section{Author contributions}

All authors have contributed to the design of the registry and/or the preparation of the manuscript. EMF was responsible for the analysis of data. ATC and PB drafted the first version of the manuscript and the remaining authors made substantial revisions to the manuscript. All authors have approved the version to be published. Apart from the selection of the countries, all design aspects were decided by the scientific Steering Committee and executed by independent Contract Research organizations.

\section{Conflicts of interest}

The members of the Steering Committee received honoraria for their advice in the planning of the Registry. Giancarlo Agnelli (GA), Rupert Bauersachs (RB), Alexander T. Cohen (ATC), Anselm K. Gitt (AKG), Patrick Mismetti (PM), Manuel Monreal (MM), Stefan N. Willich (SNW) and Peter Bramlage (PB) have received research support and/or honoraria for lectures from a number of pharmaceutical companies including Daiichi Sankyo, the sponsor of the registry. Eva-Maria Fronk (EMF), Petra Laeis (PL) and Wolf-Peter Wolf (WPW) are employees of Daiichi Sankyo Europe GmbH. The members of the Steering Committee received honoraria and travel reimbursements from Daiichi Sankyo Europe $\mathrm{GmbH}$ for their participation in Steering Committee Meetings. 


\section{References}

1. Heit JA. The epidemiology of venous thromboembolism in the community. Arterioscl Thromb Vasc Biol 2008; 28: 370-372.

2. Cohen AT, Agnelli G, Anderson FA, et al. Venous thromboembolism (VTE) in Europe. The number of VTE events and associated morbidity and mortality. Thromb Haemost 2007; 98: 756-764.

3. Suarez Fernandez C, Gonzalez-Fajardo JA, Monreal Bosch M, et al. Computerized registry of patients with thromboembolic disease in Spain (RIETE): background, objectives, methods, and preliminary results. Rev Clin Espan 2003; 203: 68-73.

4. Hach-Wunderle V, Bauersachs R, Gerlach HE, et al. Post-thrombotic syndrome 3 years after deep venous thrombosis in the Thrombosis and Pulmonary Embolism in Out-Patients (TULIPA) PLUS Registry. J Vasc Surg Venous Lymph Dis 2013; 1: 5-12.

5. Agnelli G, Gitt AK, Bauersachs R, et al. The management of acute venous thromboembolism in clinical practice - study rationale and protocol of the European PREFER in VTE Registry. Thrombosis J 2015; 13: 41.

6. Pisters R, Lane DA, Nieuwlaat R, et al. A novel user-friendly score (HAS-BLED) to assess 1-year risk of major bleeding in patients with atrial fibrillation: the Euro Heart Survey. Chest 2010; 138: 1093-1100.

7. Schulman S, Kearon C, Subcommittee on Control of Anticoagulation of the S, et al. Definition of major bleeding in clinical investigations of antihemostatic medicinal products in non-surgical patients. J Thromb Haemost 2005; 3: 692-694.

8. van Es N, Coppens M, Schulman S, et al. Direct oral anticoagulants compared with vitamin $\mathrm{K}$ antagonists for acute venous thromboembolism: evidence from phase 3 trials. Blood 2014; 124: 1968-1975.

9. Heit JA. Venous thromboembolism epidemiology: implications for prevention and management. Semin Thromb Hemost 2002; 28 (Suppl 2): 3-13.

10. Martinez C, Cohen AT, Bamber L, et al. Epidemiology of first and recurrent venous thromboembolism: a population-based cohort study in patients without active cancer. Thromb Haemost 2014; 112: 255-263.

11. Schulman S, Kakkar AK, Goldhaber SZ, et al. Treatment of acute venous thromboembolism with dabigatran or warfarin and pooled analysis. Circulation 2014 129: 764-772.

12. Mason C. Venous thromboembolism: a chronic illness. J Cardiovasc Nurs 2009; 24 (6 Suppl): S4-7.

13. Maestre A, Sanchez R, Rosa V, et al. Clinical characteristics and outcome of inpatients versus outpatients with venous thromboembolism: findings from the RIETE Registry. Eur J Intern Med 2010; 21: 377-382.

14. Tri-london.ac.uk. GARFIELD-VTE Registry Thrombosis Research Insitute, Providing Excellence in Thrombosis Research. Available at: http://www.trilondon.ac.uk/garfield-vte Accessed May 27, 2015.

15. Miniati M, Cenci C, Monti S, et al. Clinical presentation of acute pulmonary embolism: survey of 800 cases. PloS one 2012; 7: e30891.

16. Pollack CV, Schreiber D, Goldhaber SZ, et al. Clinical characteristics, management, and outcomes of patients diagnosed with acute pulmonary embolism in the emergency department: initial report of EMPEROR (Multicenter Emergency Medicine Pulmonary Embolism in the Real World Registry). J Am Coll Cardiol 2011; 57: 700-706.

17. Kearon C, Akl EA, Ornelas J, et al. Antithrombotic therapy for vte disease: Chest guideline and expert panel report. Chest 2016; 149: 315-352.

18. Di Nisio M, Raskob G, Buller HR, et al. Prediction of major and clinically relevant bleeding in patients with VTE treated with edoxaban or vitamin K antagonists. Thromb Haemost 2017; 117: 794-793.

19. Kirchhof P, Benussi S, Kotecha D, et al. 2016 ESC Guidelines for the management of atrial fibrillation developed in collaboration with EACTS. Eur Heart J 2016; 37: 2893-2962.

20. Kooiman J, van Hagen N, Iglesias Del Sol A, et al. The HAS-BLED Score Identifies Patients with Acute Venous Thromboembolism at High Risk of Major Bleeding Complications during the First Six Months of Anticoagulant Treatment. PloS one 2015; 10: e0122520.

21. Riva N, Bellesini M, Di Minno MN, et al. Poor predictive value of contemporary bleeding risk scores during long-term treatment of venous thromboembolism. A multicentre retrospective cohort study. Thromb Haemost 2014; 112: 511-521.

22. Klok FA, Hosel V, Clemens A, et al. Prediction of bleeding events in patients with venous thromboembolism on stable anticoagulation treatment. Eur Resp J 2016; 48: 1369-1376.

23. Kirchhof P, Ammentorp B, Darius H, et al. Management of atrial fibrillation in seven European countries after the publication of the 2010 ESC Guidelines on atrial fibrillation: primary results of the PREvention oF thromboemolic events-European Registry in Atrial Fibrillation (PREFER in AF). Europace 2014; 16: 6-14.

24. Hokusai VTE Investigators, Buller HR, Decousus $\mathrm{H}$, et al. Edoxaban versus warfarin for the treatment of symptomatic venous thromboembolism. N Engl J Med 2013; 369: 1406-1415.

25. Schulman S, Kearon C, Kakkar AK, et al. Dabigatran versus warfarin in the treatment of acute venous thromboembolism. N Engl J Med 2009; 361: 2342-2352.

26. Investigators $\mathrm{E}$, Bauersachs R, Berkowitz SD, et al. Oral rivaroxaban for symptomatic venous thromboembolism. N Engl J Med 2010; 363: 2499-2510.

27. Investigators E-P, Buller HR, Prins MH, et al. Oral rivaroxaban for the treatment of symptomatic pulmonary embolism. N Engl J Med 2012; 366: 1287-1297.

28. Agnelli G, Buller HR, Cohen A, et al. Oral apixaban for the treatment of acute venous thromboembolism. N Engl J Med 2013; 369: 799-808.

29. van der Hulle T, Kooiman J, den Exter PL, et al. Effectiveness and safety of novel oral anticoagulants as compared with vitamin $\mathrm{K}$ antagonists in the treatment of acute symptomatic venous thromboembolism: a systematic review and metaanalysis. J Thromb Haemost 2014; 12: 320-328.

30. Becattini C, Agnelli G, Prandoni P, et al. A prospective study on cardiovascular events after acute pulmonary embolism. Eur Heart J 2005; 26: 77-83.

31. Sorensen HT, Horvath-Puho E, Pedersen L, et al. Venous thromboembolism and subsequent hospitalisation due to acute arterial cardiovascular events: a 20-year cohort study. Lancet 2007; 370: 1773-1779. 